\title{
A new approach for accurate quantitative determination using fluorine nuclear magnetic resonance spectroscopy
}

\author{
Taichi Yamazaki ${ }^{*}$, Takeshi Saito and Toshihide Ihara
}

\author{
National Metrology Institute of Japan, National Institute of Advanced Industrial Science and \\ Technology (AIST), 1-1-1 Umezono, Tsukuba, Ibaraki 305-8563, Japan
}

(Received March, 16, 2017; Revised March, 28, 2017; Accepted March, 31,2017)

\begin{abstract}
A new approach for accurate quantitative determination by fluorine nuclear magnetic resonance $\left({ }^{19} \mathrm{~F}\right.$ NMR) spectroscopy is presented and discussed. ${ }^{19} \mathrm{~F}$ NMR spectroscopy has similar properties to ${ }^{1} \mathrm{H}$ NMR spectroscopy except for the wide chemical shift dispersion, which is the main difference between the two techniques. The off-resonance effect, which is caused by the chemical shift difference between the excitation pulse offset and the resonance signal position, is expected to be the most important parameter influencing the accuracy of the quantitative analysis by ${ }^{19} \mathrm{~F}$ NMR spectroscopy. We propose a new method that reduces the influence of the off-resonance effect in ${ }^{19} \mathrm{~F}$ NMR measurement. First, we discussed the areas of signals originating from different functional groups in a molecule. Then, we evaluated the areas of signals originating from two different reference materials (RMs). The accuracy of the ratios of two signal areas in the ${ }^{19} \mathrm{~F} \mathrm{NMR}$ spectrum of a molecule of Potassium perfluorooctanesulfonate was less than $1 \%$. We also evaluated the purity of flusulfamide and diflubenzuron as RMs, and found that the method could determine the purity to less than 1 $\%$ accuracy relative to the reference values.
\end{abstract}

Keywords: qNMR, ${ }^{19}$ F NMR, Internal standard method, Purity, off resonance effect . C 2017 ACG Publications. All rights reserved.

\section{Introduction}

In conventional methods for quantitative analysis, such as chromatography, a reference material (RM) of a target analyte is essential to obtain accurate quantitative results. In contrast, in the quantitative NMR technique, an RM that differs from the target analyte is used [1]. Characterized by this unique property, the application of the quantitative NMR technique to the direct analysis of potency or the purity determination of RMs has attracted much interest. Several papers have been published on the quantitative analysis by ${ }^{1} \mathrm{H}$ NMR spectroscopy [2-4]. As it can be used not only for the purity determination of a pure material [5-7] but also for the concentration evaluation [8-10], this technique is a high-utility analytical method. Discussions of the accuracy of quantitative ${ }^{1} \mathrm{H}$ NMR spectroscopy in purity determination have also emerged [5,11,12].

${ }^{19} \mathrm{~F}$ NMR spectroscopy has similar properties to ${ }^{1} \mathrm{H}$ NMR spectroscopy; the two techniques have identical nuclear spin and similar resonance frequency and sensitivity. The relative isotope abundance is $100 \%$ [13]. However, unlike ${ }^{1} \mathrm{H}$ nuclei, ${ }^{19} \mathrm{~F}$ is structurally isolated in many cases. Based on these facts, quantitative ${ }^{19} \mathrm{~F}$ NMR spectroscopy is considered to be a useful analytical method. Organic fluorine compounds are high-functionality compounds used in various fields, and they are attractive as

\footnotetext{
* Corresponding author E-Mail: t-yamazaki@aist.go.jp

The article was published by Academy of Chemistry of Globe Publications www.acgpubs.org/jcm (C) Published 03/31/2017 EISSN:1307-6183

http://doi.org/10.25135/jcm.3.17.03.036
} 
an analyte [14]. Some applications have been reported $[15,16]$. However, the precision of the results, such as repeatability, is mainly discussed in those papers, and as far as we know, there are no reports discussing in detail the methodology for accurate analysis. The main difference between ${ }^{19} \mathrm{~F}$ NMR spectroscopy and ${ }^{1} \mathrm{H}$ NMR spectroscopy is the chemical shift dispersion. The chemical shifts of the resonance signals in the ${ }^{19} \mathrm{~F}$ NMR spectra are sensitive to their surrounding structures. Due to the wide chemical shift dispersion, an excitation pulse cannot produce a uniform excitation throughout the chemical shift region of interest. In this regard, the relative position between a resonance signal and an excitation pulse offset is an important factor for accurate quantitative ${ }^{19} \mathrm{~F}$ NMR analysis.

In this paper, we discuss ${ }^{19} \mathrm{~F}$ NMR measurement parameters and the off-resonance effect, which may cause bias in the excited signal area. Based on this knowledge, we present a new approach for accurate quantitative determination by ${ }^{19} \mathrm{~F}$ NMR spectroscopy. A comparison of the areas of signals originating from ${ }^{19} \mathrm{~F}$ in different regions of a given molecule was carried out to optimize the measurement parameters. The parameters were then applied to the quantitative analysis of ${ }^{19} \mathrm{~F}$ in different compounds.

\section{Experimental}

\subsection{Apparatus and parameters}

${ }^{19} \mathrm{~F}$ NMR spectra were obtained with a Varian VNS 600 spectrometer operating at the ${ }^{19} \mathrm{~F}$ resonance frequency of $564.43 \mathrm{MHz}$. The probe was a Varian ${ }^{15} \mathrm{~N}-{ }^{31} \mathrm{P} /{ }^{1} \mathrm{H}-{ }^{19} \mathrm{~F}$ dual-broadband probe equipped with a pulsed field gradient (PFG). Temperature was regulated at $25{ }^{\circ} \mathrm{C}$. A typical set of ${ }^{19} \mathrm{~F}$ NMR experimental parameters was as follows: $131579.0 \mathrm{~Hz}(233.1 \mathrm{ppm})$ spectral width, $1.0 \mathrm{~s}$ acquisition time, $13.0 \mu \mathrm{s}\left(90^{\circ}\right)$ or $4.3 \mu \mathrm{s}\left(30^{\circ}\right)$ pulse width, and $60 \mathrm{~s}$ relaxation delay, and 32 scans were acquired. The inversion recovery pulse sequence was used for longitudinal relaxation time $\left(T_{1}\right)$ determination. Data processing was performed using MestReNova ver.6.1.1. All signals were integrated to the range of approximately 80 times of the full-width at half maximum (FWHM) of the integral signal. The integral range was evaluated in advance. The range was optimized by the approach in which the integral area reached a plateau when expanded [17].

\subsection{Materials and preparation}

Methanol- $d_{4}, \quad$ 4-chloro- $N$-(2-chloro-4-nitrophenyl)-3-(trifluoromethyl)benzenesulfonamide (flusulfamide, $\left.(0.995 \pm 0.006) \mathrm{kg} \mathrm{kg}^{-1}\right), \quad N$-[(4-chlorophenyl)carbamoyl]-2,6-difluorobenzamide (diflubenzuron, $\left.(0.998 \pm 0.008) \mathrm{kg} \mathrm{kg}^{-1}\right)$, and 4-bromo-2-(4-chlorophenyl)-1-(ethoxymethyl)-5(trifluoromethyl)-1 $H$-pyrrole-3-carbonitrile (chlorfenapyr, $\left.(0.996 \pm 0.005) \mathrm{kg} \mathrm{kg}^{-1}\right)$ were obtained from Wako Pure Chemical Industries, Ltd. (Wako). Potassium perfluorooctanesulfonate (PFOS-K) was obtained from Wellington Laboratories Inc. Flusulfamide, diflubenzuron, and chlorfenapyr are RMs, and their chemical purities have been certified.

For the intramolecular analysis, PFOS-K was dissolved in methanol- $d_{4}$ to make a concentration of approximately $5 \mathrm{mg} \mathrm{g}^{-1}$. For the intermolecular analysis, chlorfenapyr was used as the internal standard (IS) to determine the purities of flusulfamide and diflubenzuron as analytes. Both the analytes and IS were weighed accurately on aluminum pans in an ultra-micro balance (UMX2, METTLER TOLEDO), added to a vial, and dissolved in methanol- $d_{4}$. All the concentrations were approximately 5 $\mathrm{mg} \mathrm{g}^{-1}$.

\subsection{Optimization of parameters for quantitative ${ }^{19} \mathrm{~F}$ NMR measurement with PFOS-K}

All the parameters for quantitative ${ }^{19} \mathrm{~F}$ NMR analysis were optimized with reference to those for quantitative ${ }^{1} \mathrm{H}$ NMR analysis $[5,11,12] . T_{1}$ of ${ }^{19} \mathrm{~F}$ signals in PFOS-K was measured to determine the relaxation delay. The repeatability of the signal areas with different pulse flip angles of $90^{\circ}$ and $30^{\circ}$ was evaluated. The signal area depend on the chemical shift difference between the excitation pulse offset and the resonance frequencies was evaluated by comparing the spectral areas observed with different excitation pulse offset values. The offset was changed at an interval of $10 \mathrm{ppm}$ to cover the 
entire spectral width. In addition, the offset was changed at an interval of $1 \mathrm{ppm}$ near the analyte signals. The ratio of two different PFOS-K signal areas was used to optimize the quantitative ${ }^{19} \mathrm{~F} N M R$ measurement conditions.

\subsection{Application of ${ }^{19}$ F NMR spectroscopy to intermolecular analysis}

The purity of flusulfamide and diflubenzuron was determined from chlorfenapyl (IS) by ${ }^{19} \mathrm{~F}$ NMR measurement under the optimum conditions. The results obtained were compared to the purity of the RMs.

\section{Results and discussion}

\subsection{Optimization of parameters for quantitative ${ }^{19} \mathrm{~F}$ NMR measurement}
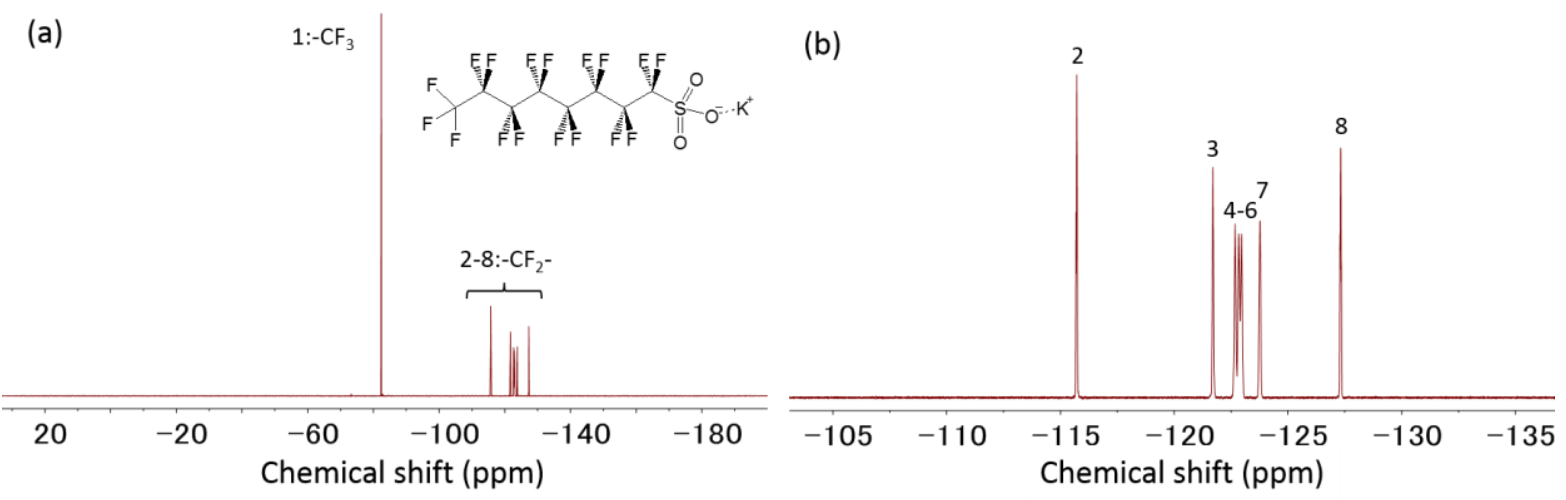

Figure 1. ${ }^{19} \mathrm{~F}$ NMR spectrum of PFOS-K. (a) The full spectrum measured in this work. (b) Expansion of the spectral region of fluorine in main chain $\left(-\mathrm{CF}_{2}-\right)$.

A typical ${ }^{19} \mathrm{~F}$ NMR spectrum of PFOS-K is shown in Fig. 1. The ${ }^{19} \mathrm{~F}$ NMR signals indicated wide chemical shift dispersion compared to the ${ }^{1} \mathrm{H}$ NMR signals. Although PFOS-K is composed of a fluorinated alkyl chain, the signals were well separated, which indicates the wide chemical shift dispersion in ${ }^{19} \mathrm{~F}$ NMR measurement. In quantitative NMR measurement, signal separation is one of the most important factors because if the signals were not separated from each other, it would not be possible to evaluate their areas independently. Resonance overlap is often a source of problem in ${ }^{1} \mathrm{H}$ NMR measurement. Therefore, ${ }^{19} \mathrm{~F}$ NMR measurement may offer a way to counter the problem.

$T_{1}$ of PFOS-K was estimated to determine the relaxation delay in quantitative ${ }^{19} \mathrm{~F}$ NMR measurement. $T_{1}$ was approximately $2.2 \mathrm{~s}$ for a terminal $\mathrm{CF}_{3}$ resonance, and between $1.0 \mathrm{~s}$ and $1.9 \mathrm{~s}$ for internal $\mathrm{CF}_{2}$ resonances. Based on this result, approximately $30 \mathrm{~s}$ is sufficient as the relaxation delay in theory. Nevertheless, we set the relaxation delay at $60 \mathrm{~s}$ in the quantitative ${ }^{19} \mathrm{~F}$ NMR experiments of PFOS-K, so that we could completely ignore the influence of the relaxation delay. As a free induction decay signal relaxes to noise level, an acquisition time of $1 \mathrm{~s}$ was used.

The smaller the excitation pulse width is, the wider the excitation field range is. Therefore, $90^{\circ}$ and $30^{\circ}$ excitation pulses were used to evaluate different excitation profiles. Note that the chemical shift of the terminal $\mathrm{CF}_{3}$ signal was considerably different from those of the $\mathrm{CF}_{2}$ signals. The areas of the two signals from a signal resonated at signal 1 and signal 8 in Fig. 1, respectively, compared with a variety of excitation pulse offsets. The bias from the expected ratio of the areas of signals 1 and 8 was approximately $10 \%$ and $2 \%$ when $90^{\circ}$ and $30^{\circ}$ pulses were used, respectively. A smaller pulse width is expected to give a wider flat excitation field profile than a larger pulse width, and our results agree with this idea. Signals 1 and 8 are approximately $50 \mathrm{ppm}$ apart, and the off-resonance effect becomes the major factor for obtaining accurate NMR areas. 

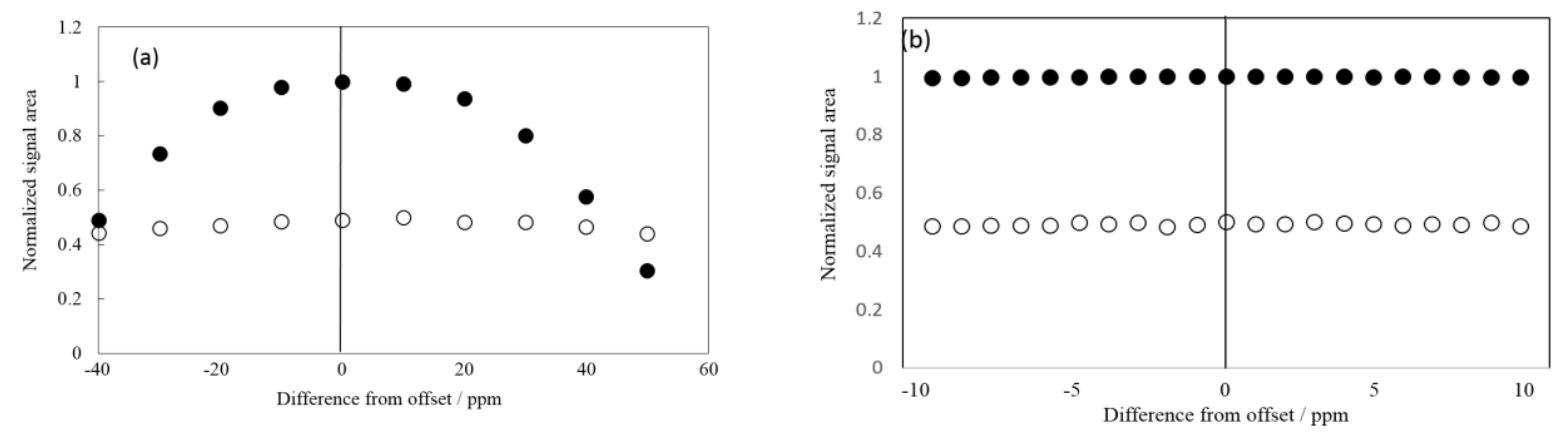

Figure 2. Normalized signal area of PFOS-K at different excitation pulse offsets. White circles $(\bigcirc)$ show results of measurement using the $30^{\circ}$ pulse. Black circles $(\mathbf{O})$ show results of measurement using the $90^{\circ}$ pulse. (a) The offset was changed from the lowest field to the highest one in steps of 10 ppm. (b) The offset was changed from the lowest field to the highest one in steps of $1 \mathrm{ppm}$.

As the chemical shift dispersion in the ${ }^{1} \mathrm{H}$ NMR spectrum is much narrower, this effect is not a problem. However, the off-resonance effect on the quantitative ${ }^{19} \mathrm{~F}$ NMR value has not been evaluated before. Thus, the relationship between the normalized area of signal 1 in PFOS-K and the chemical shift difference between the analyte resonance and the offset was evaluated using $90^{\circ}$ and $30^{\circ}$ pulses. The result of the offset array at signal 1 is shown in Fig. 2. Figure 2 (a) indicates that when the offset was approximately $40 \mathrm{ppm}$ from the analyte signal, the signal area was reduced to approximately $50 \%$ of its expected area in the case of the $90^{\circ}$ pulse. Although the influence of the off-resonance effect using the $30^{\circ}$ pulse was much less than that using the $90^{\circ}$ pulse, the data showed much more scatter due to poor repeatability. The influence of the off-resonance effect in a narrow field range (approximately $20 \mathrm{ppm}$ ) was insignificant to the measurement variation. Although this effect influence to bias of between signals, but it could be ignored if the measurement variation is larger than this influence. When high accuracy is discussed with high measurement repeatability, it might be needed to consider as uncertainty factor. This is because the repeatability of the signal area depends on the signal-to-noise ratio $(\mathrm{S} / \mathrm{N})$ of the signal, and $\mathrm{S} / \mathrm{N}$ can be improved by increasing the number of scans. The repeatability of signal 1 using the $90^{\circ}$ pulse was considerably better than that using the $30^{\circ}$ pulse when identical scans were compared. With 32 scans, the repeatability of the former case was less than $0.1 \%$ whereas that of the latter case was more than $0.2 \%$. Although the off-resonance effect was smaller, the result obtained using the $30^{\circ}$ pulse indicated that further treatments were required to achieve an accuracy of $1 \%$. Use of the $90^{\circ}$ pulse is preferable to obtain a better quality spectrum within a shorter period of time.

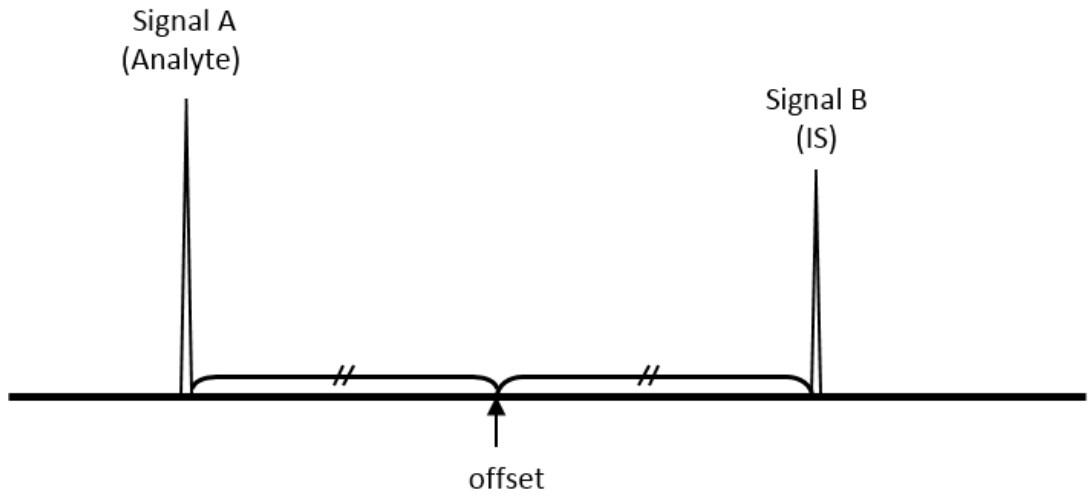

Figure 3. Setting the offset for the new method overcomes the off-resonance effect 
It is important to determine the measurement conditions that would suppress the influence of the off-resonance effect to a minimum for accurate quantitative ${ }^{19} \mathrm{~F}$ NMR measurements. Inspection of Fig. 2 revealed that the magnitude of the signal area off-resonance effect showed a symmetric behavior to the excitation pulse offset. When the offset was set midway between the analyte signal and the IS signal (Fig. 3), both signal intensities should be influenced by an identical fraction of bias. Therefore, the influence of the off-resonance effect on the signal area is completely suppressed, and it is expected that the signal area will be obtained with high accuracy. It is concluded that an excitation pulse should be applied midway between the two resonance signals whose areas are evaluated. This is the most important factor for obtaining an accurate ratio of two signals in ${ }^{19} \mathrm{~F}$ NMR measurement. This method can therefore be used for accurate quantitative NMR measurement targeting two signals with a large chemical shift difference.

\subsection{Method validation with quantitative analysis of intramolecular signals in PFOS-K}

\begin{tabular}{ll} 
Table 1. Optimum parameters for ${ }^{19} \mathrm{~F}$ NMR measurement. \\
\hline Measurement nucleus & $:{ }^{19} \mathrm{~F}$ \\
Temperature & $: 25^{\circ} \mathrm{C}$ \\
Spectral width & $: 131579.0 \mathrm{~Hz}(233.1 \mathrm{ppm})$ \\
Relaxation delay & $: 60 \mathrm{~s}$ \\
Acquisition time & $: 1 \mathrm{~s}$ \\
Pulse angle & $: 30^{\circ}$ or $90^{\circ}$ \\
Number of scans & $: 32$ \\
\hline
\end{tabular}

The optimum parameters for the quantitative ${ }^{19} \mathrm{~F}$ NMR measurement of PFOS-K are shown in Table 1. In the proposed method, two resonance signals are the target of measurement and the excitation pulse offset is set midway between the two resonances. The difference between the two signals was determined based on the signal in the lower field region. Quantitative ${ }^{19} \mathrm{~F}$ NMR measurement was carried out and the signal areas were divided by the number of fluorine atoms. The results are shown in Table 2.

The difference of two signal areas from the experiments was reduced to $1 \%$ or less using the proposed method, indicating that the influence of the off-resonance effect is decreased in this method. Moreover, the deviation among the results obtained using the $90^{\circ}$ pulse was smaller than that using the $30^{\circ}$ pulse as $\mathrm{S} / \mathrm{N}$ using the $90^{\circ}$ pulse was much higher. Therefore, use of the $90^{\circ}$ pulse is advantageous for accurate quantification with ${ }^{19} \mathrm{~F}$ NMR spectroscopy. Although the influence of the off-resonance effect using the $90^{\circ}$ pulse was significant, the effect was almost suppressed and could be ignored using this method.

Table 2. Estimation of off-resonance effect by comparing intramolecular signal areas. Differences were evaluated based on the signal in the lower field.

\begin{tabular}{cccccc}
\hline \multicolumn{2}{c}{ Comparison signal } & \multicolumn{2}{c}{$90^{\circ}$} & \multicolumn{2}{c}{$30^{\circ}$} \\
\hline \multirow{3}{*}{$\mathrm{A}$} & $\mathrm{B}$ & \multicolumn{2}{c}{$\begin{array}{c}\text { Difference over area of signal A } \\
/ \%\end{array}$} & \multicolumn{2}{c}{$\begin{array}{c}\text { Difference over area of signal A } \\
/ \%\end{array}$} \\
\cline { 3 - 6 } & & New method* & Offset to -85 ppm & New method* & Offset to -85 ppm \\
\hline 1 & 2 & -0.86 & -3.00 & 1.40 & 0.43 \\
1 & 3 & -0.88 & -5.77 & 0.50 & -0.16 \\
1 & $4-6$ & 0.19 & -8.20 & -0.55 & -2.09 \\
1 & 7 & -0.14 & -9.94 & -0.53 & -1.79 \\
2 & 8 & -0.81 & -7.16 & 1.42 & -2.21 \\
\hline
\end{tabular}

*The offset is set midway between the two signals. 


\subsection{Application to intermolecular analysis}

Finally, quantitative analysis of two molecules was attempted using the new method. The excitation pulse offset was set midway between the IS signal and the analyte signal. The NMR spectra of mixtures of flusulfamide and chlorfenapyl, and diflubenzuron and chlorfenapyl are shown in Fig. 4 (a) and (b), respectively. In both cases, chlorfenapyl was the IS. In Fig. 4 (a), the signals of flusulfamide and IS were approximately $6.5 \mathrm{ppm}$ apart, which is considered to be a small chemical shift difference. In contrast, in Fig. 4 (b), the signal of diflubenzuron was separated from that of IS by approximately $57 \mathrm{ppm}$. Due to the large chemical shift difference between the two signals in the latter case, an accurate ratio of the two signals would not be obtained under conventional quantitative measurement conditions.

(a)

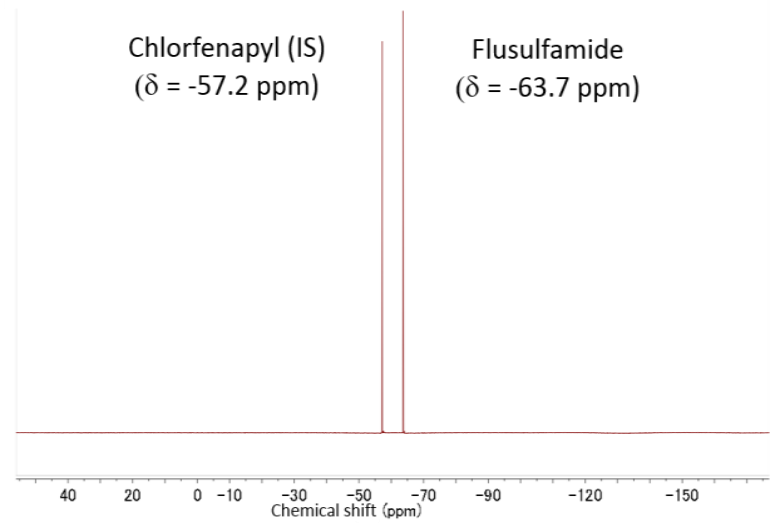

(b)

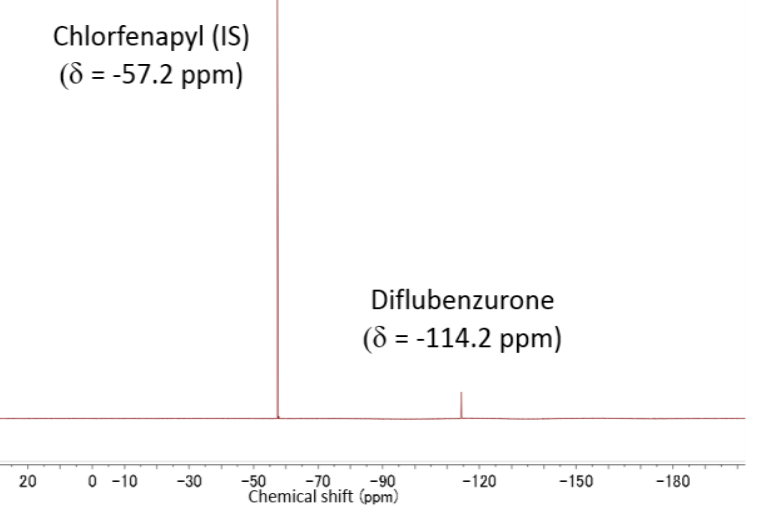

Figure 4. (a) ${ }^{19} \mathrm{~F}$ NMR spectra of mixture of flusulfamide (analyte) and chlorfenapyl (IS) for intermolecular analysis. (b) ${ }^{19}$ F NMR spectra of mixture of diflubenzuron (analyte) and chlorfenapyl (IS) for intermolecular analysis.

Although the prepared concentrations were almost identical and the signal areas were similar, the signal intensity of diflubenzuron was remarkably lower than that of IS.

The purity obtained by the proposed method is shown in Table 3 . All measurement results are in good agreement with the reference values. The proposed method was proven to yield an accurate ratio of the two signals, or the purity, even if the signals were separated widely. It is concluded that the proposed method can be used to determine the purity of organic fluorine compounds with an accuracy of $0 \%$ to $1 \%$ of the reference values by quantitative ${ }^{19} \mathrm{~F}$ NMR measurement.

Table 3. Results of intermolecular analysis

\begin{tabular}{|c|c|c|c|}
\hline \multirow{2}{*}{ Analyte } & \multicolumn{2}{|c|}{ Quantitative value $\left(\mathrm{kg} \mathrm{kg}^{-1}\right)$} & \multirow{2}{*}{$\begin{array}{l}\text { Reference value } \\
\qquad\left(\mathrm{kg} \mathrm{kg}^{-1}\right)\end{array}$} \\
\hline & $90^{\circ}$ pulse & $30^{\circ}$ pulse & \\
\hline Flusulfamide & $0.996(0.002)$ & $1.001(0.008)$ & $0.995(0.006)$ \\
\hline Diflubenzuron & $0.997(0.005)$ & $1.012(0.009)$ & $0.998(0.008)$ \\
\hline
\end{tabular}

Number in parentheses is the standard deviation.

\section{Conclusions}

We propose a new method for obtaining the accurate ratio of two single areas by ${ }^{19} \mathrm{~F}$ NMR measurement. In ${ }^{19} \mathrm{~F}$ NMR measurement, the wide chemical shift dispersion is advantageous for structure determination, but is a big disadvantage for the accurate quantification of signal areas. Our new method solves the problems caused by the off-resonance effect. The developed method reduced the influence of the off-resonance effect. The application of this method is not limited to ${ }^{19} \mathrm{~F}$ NMR measurement, but can be extended to ${ }^{13} \mathrm{C}$ NMR, ${ }^{31} \mathrm{P}$ NMR, or other nuclei when the chemical shift difference of two target signals is large. Under the optimum conditions, the ratio of the two signal 
areas positioned more than $50 \mathrm{ppm}$ apart can be obtained within an accuracy of $\pm 1 \%$. By using this method, accurate ${ }^{19} \mathrm{~F}$ NMR quantitative measurement can be carried out. This method can expand the measurement capability of quantitative NMR. Moreover, with the accurate measurement conditions of ${ }^{19} \mathrm{~F}$ NMR set, it is now possible to cross check the purity results obtained by ${ }^{19} \mathrm{~F}$ and ${ }^{1} \mathrm{H}$ NMR measurement of fluorine- and proton-containing molecules.

\section{References}

[1] H. Janche (1998). NMR spectroscopy as a primary analytical method, CCQM Report, 98(2)

[2] P. Giraudeau, I. Tea, G. S. Remaud and S. Akoka (2014). Reference and normalization methods: Essential tools for the intercomparison of NMR spectra, J. Pharm. Biomed Anal., 93, 3-16.

[3] C. Simmler, J. G. Napolitano, J. B. McAlpine, S. N. Chen and G. F. Pauli (2014). Universal quantitative NMR analysis of complex natural samples, Current opinion in biotechnology, 25, 51-59.

[4] G. A. Barding, R. Salditos and C. K. Larive (2012). Quantitative NMR for bioanalysis and metabolomics, Anal. Bioanal. Chem., 404(4), 1165-1179.

[5] T. Yamazaki and A. Takatsu (2014). Quantitative NMR spectroscopy for accurate purity determination of amino acids, and uncertainty evaluation for different signals, Accred. Qual. Assur., 19, 275-282.

[6] S. Mahajan and I. P. Singh (2013). Determining and reporting purity of organic molecules: why qNMR, Magn. Reson. Chem., 51, 76-81.

[7] T. Schoenberger (2012). Determination of standard sample purity using the high-precision H-1-NMR process, Anal. Bioanal. Chem., 403(1), 247-254.

[8] X. Liang, L. Du, F. Su, H. S. Parekh and W. Su (2014). The application of quantitative NMR for the facile, rapid and reliable determination of clindamycin phosphate in a conventional table formulation, Magn. Reson. Chem., 52(4), 178-182.

[9] H. Gadape and K. Parikh (2011). Quantitative determination and validation of Carvedilol in pharmaceuticals using quantitative nuclear magnetic resonance spectroscopy, Analytical methods, 3(10), 2341-2347.

[10] I. W. Burton, W., M. A. Quilliam and J. A. Walter (2005). Quantitative H-1 NMR with external standards: Use in preparation of calibration solutions for algal toxins and other natural products, Anal. Chem., 77, 3123-3131.

[11] M. Weber, C. Hellriegel, A. Rueck, R. Sauermoser and J. Wuethrich (2013). Using high-performance quantitative NMR (HP-qNMR(A (R))) for certifying traceable and higly accurate purity values of organic reference materials with uncertainties < $0.1 \%$, Accred. Qual. Assur., 18(2), 91-98.

[12] T. Saito, S. Nakaie, M. Kinoshita, T. Ihara, S. Kinugasa, A. Nomura and T. Maeda (2004). Practical guide for accurate quantitative solution state NMR analysis, Metrologia, 41, 213-218.

[13] M. Berglund and M. Wieser (2011). Isotope compositions of the elements 2009 (IUPAC Technical Report), Pure Appl. Chem., 83(2), 397-410.

[14] F. Babudri, G. M. Farinola, F. Naso and R. Ragni (2007). Fluorinated organic materials for electronic and optoelectronic applications: the role of the fluorine atom, Chem. Commun., 1003-1022.

[15] A. Mutlib, R. Espina, J. Atherton, J. Wang, R. Talaat, J. Scatina and A. Chandrasekaran (2012). Alternate Strategies to Obtain Mass Balance without the Use of Radio labeled Compounds: Application of Quantitative Fluorine (F-19) Nuclear Magnetic Resonance (NMR) Spectroscopy in Metabolism Studies, Chem. Res. Toxicol., 25(3), 572-583.

[16] N. M. Do, M. A. Oliver, J. J. Salisbury and C. B. Wager (2011). Application of quantitative F-19 and H-1 NMR for reaction monitoring and in situ yield determinations for an early stage pharmaceutical candidate, Anal. Chem., 83(22), 8766-8771.

[17] T. Yamazaki, T. Saito, T. Miura and T. Ihara (2012). Investigation of analysis conditions for accurate quantitative NMR analysis, BUNSEKIKAGAKU, 61(11), 963-967.

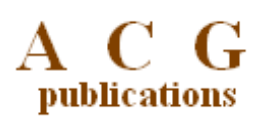

(C) 2017 ACG Publications 